\title{
Development of a risk assessment system based on pattern matching of behavioural fault models
}

\author{
M. Costescu ${ }^{1}$, E. Doval ${ }^{2} \&$ S. Kovacs ${ }^{1}$ \\ ${ }^{I}$ INCDPM "Alexandru Darabont", Romania \\ 2 "Spiru Haret” University, Romania
}

\begin{abstract}
Accidents are often repeated. Not learning from past accidents leads towards new ones. The larger part of occupational accidents are caused by the mistakes of human operators, by their mis-judgements and negligence. Behavioural fault models could describe, on the basis of accident and incident experience, the occurence of an accident by the mistakes made by the operator, putting into balance the initial causes of the mistaken actions (lack of knowledge, trying to shortcut longer but safer procedures, etc.) and the events that occured because of these causes. Behavioural fault models are perfectly able to be developed using ontologies, an assessment system based on past knowledge, and driven by ontologies could be very usefull to judge the safety at a workplace. The paper describes our research in developing such an ontological driven safety assessment system prototype and also the obtained results of running this prototype in Romanian small and medium enterprises. The system starts with the building of a behavioural normal activity model-specific, for example, for the activity of work at heights. By pattern matching this model with behavioural fault models developed from past experiences and also by direct observation of the workplace a quantified degree of safety could be established.
\end{abstract}

Keywords: behavioural fault models, risk assessment, human operator.

\section{Introduction}

Material loss, incidents and accidents at the workplace have almost always the same cause, the same mode of manifestation and the same pre-event warning 
which are almost always bypassed. Accidents happen every day and few people or organisations care or learn something from the accident. A number of accidents that have occurred after the same pattern in Romania and abroad gave us the basis of our research, to see if there were similar fault behaviour patterns of the human operators that were guilty of the accident occurrence. If so, is there any possibility to define a human behavioural fault model which could be used in order to assess eventual incidents because of improper behaviour? In our research we found that such models could be built on specific activities and are extremely interesting to be developed as predictors for future troubles; also they could be used as case studies for efficient safety training. By pattern matching real behaviour with the models an efficient risk assessment could be performed by the human operator's point of view.

\section{Humans and loss}

Information regarding work can be systematically obtained about worker dependant factors that trigger undesired events (be it loss, incident or accident), the physiologically and psychologically worker condition at the start/end of work. Also, it is possible to obtain information about pre-accident states, causes of accidents, conditions of their occurrence, erroneous actions and measures of preventing them in a systematic matter [1]. As known, humans are the main accident perpetrators. About $75 \%$ of the occupational accidents that occurred in Romania [2] had people as the main cause of the accidents.

Human errors are modeled by behavioural models [3] which could start from very simple ones based on active (deliberative) and reactive behaviours. The start-up of such research was done in the military field [4]. Later developments used such models in order to design various societal strategies [5]. Generally such models are qualitative ones and are connected especially with the problem of learning [6, 7]. Literature [8] describes generally five such model categories:

- the biological model;

- the psychoanalytic model;

- the behaviourist model;

- the cognitive-behavioural model;

- the humanistic model.

The most suitable model for safety research could be the cognitivebehavioural model. Models are used mainly to describe behaviours including potential dangerous behaviours. Two prime behaviours are interesting for our workplace safety problem: reactive and active behaviours.

The reactive behaviours, reacting at an event that occurred in the work context could be categorised into four types:

- instinctive behaviour that follows a simple physical stymulus, state, reaction pattern;

- learned behaviour - instinctive behaviour within a social context;

- drive controlled behaviour - reactive behaviour triggered by a physical need; 
- emotionally controlled behaviour - reactive behaviour triggered by an emotional state.

The active behaviours are defined by objectives approached by action plans [9] These behavioural models were the basis of our human behavioural fault model (HBFM) which proposes to answer at a very simple and in the same time complicated question: where, why and when could a person make an error at the workplace? The next chapter shows the basics of the model.

\section{The human behavioural fault model}

We have built our model considering it as a qualitative one which:

- could be descriptive for repetitive incidents;

- could include fuzziness for better representation of obscure incidents;

- could serve as a framework case study against good and best practice procedures;

- are easy understandable and have distinctive causes for a certain behaviour. The schema of the model is shown in figure 1.

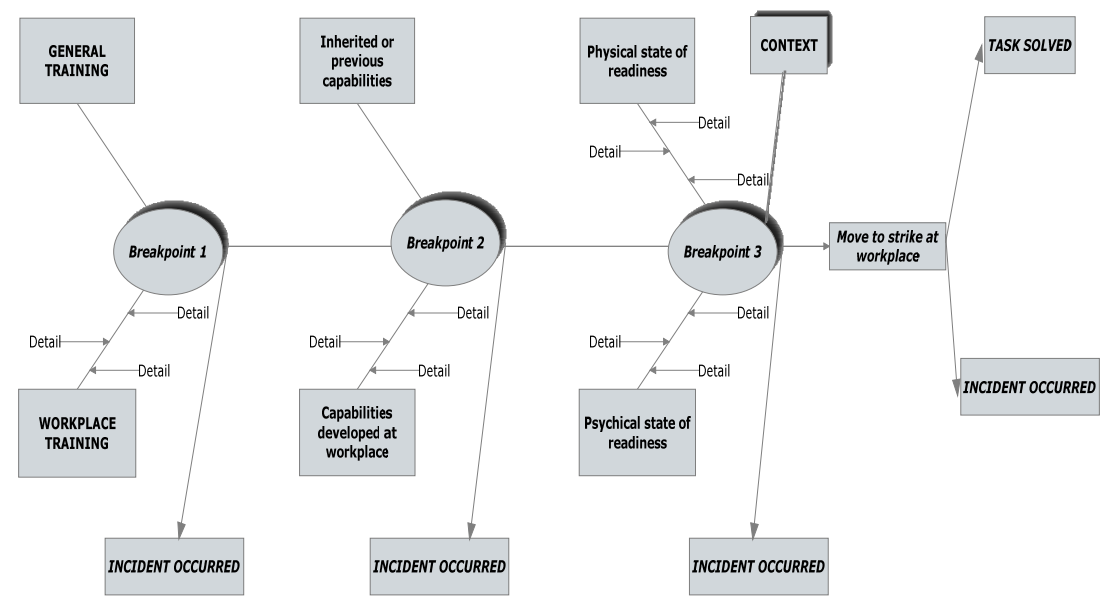

Figure 1: The human behaviour fault model general schema.

Actually, there are direct behavioural causes till the "move to strike" performed by the worker to do the designed task. Incidents could occur also in the training phase and in the development of specific capabilities.

This model takes into consideration the most general activities that could be influenced by the bad behaviour of the worker, from task reception to the elimination of the by-products. At every step:

1. the worker could perform the task correctly;

2. the worker could perform the task wrongly but no consequences occur till the next step of the activity;

3. the worker performs the task wrongly and an incident occurs which ends the process of work. 


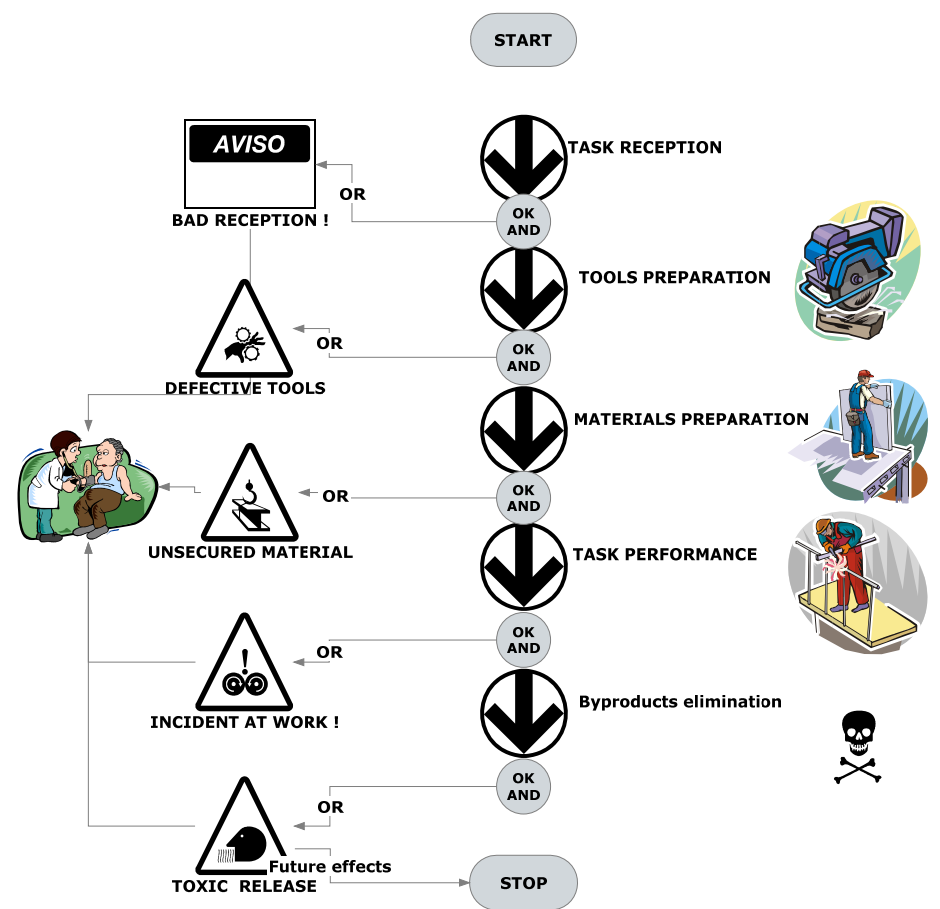

Figure 2: The "move to strike" sub model.

The model serves as a framework for specific scenarios. These scenarios or mini-models are activity specific and are actually used for assessment. Generally there is a worst case scenario in which every HBFM component is assessed at 0 (on a $0 . . .5$ scale), the worst case scenario being the equivalent of a freshman with no instruction and no capabilities for the workplace, being solicited to perform a task.

\section{Behaviour clusters-collecting and processing signs of bad behaviour at work}

Behaviour clustering is the way to collect and process the human approach optimally. An analysis agent which automatically processes behavioural clusters once collected, in the terms of human-system relationship takes into account states of knowledge and behaviour of human operators together with the system possible responsive actions. A Self-Organizing Map could be used [10] as the clustering algorithm. Micro models of behaviour, repeated continously are the most important here. They include rational choice models for decision making under uncertainty and risk as well in strategic situations and in collective decision making. Models also incorporate complex assumptions like social orientations and distributional preferences. This clustering and micro-model approach is a little time consuming, not allowing a very fast assessment. 


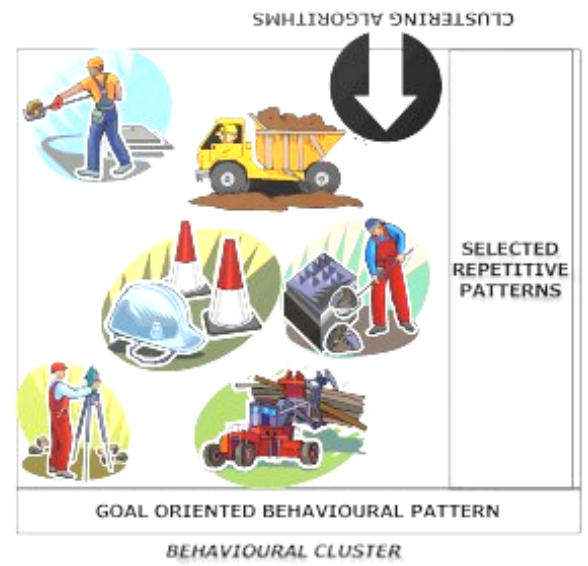

Figure 3: $\quad$ Schema of a behavioural cluster.

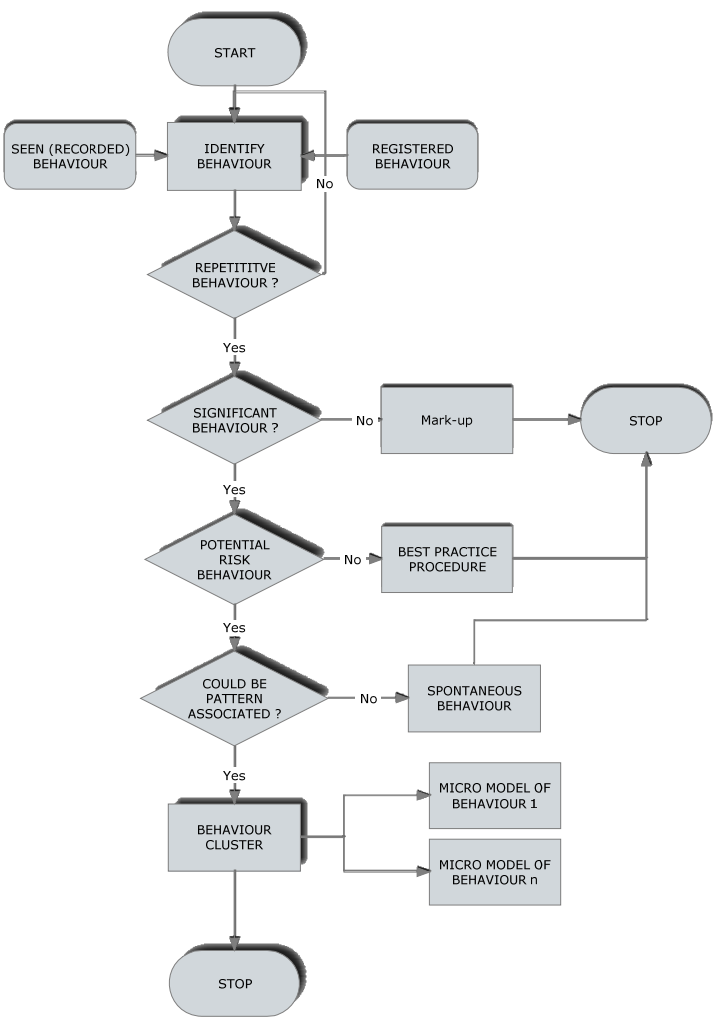

Figure 4: Behaviour processing into behavioural clusters. 
However, the repetition of bad (or good) behaviours in time is the key to a safe workplace-so the time lost collecting data could be useful later. A general schema of the behavioural cluster is prezented in figure 3 .

The schema of behaviour processing into clusters is shown in figure 4 .

The behavioural clusters are developed through repetitive observations over a period of time (usually one week) of the worker at his/hers workplace. This observation could be performed directly or through various video cameras mounted at the workplace. An additional approach would be to ask the worker to register all the movements that he/she performs in order to have a written reference that could be compared with the observed situation. The behaviour is retained if it is:

- repetitive;

- significant;

- potential risk behaviour;

- it could be associated to a pattern;

If so, the behaviour is clustered. There are many ways to do this, it could be simply a meta-file attached to the video and other registering or the behaviour cluster could be shaped as a Worker Specific Safety Use Case (WSSUC).

\section{Risk assessment using the human behaviour fault model-experiments and results}

By using the model the human factor part of risk occurrence and development into incidents and accidents could be assessed. The behaviour cluster is compared with various scenarios derived from HBFM, generally a pair of bestworst scenarios. These scenarios include unexpected events occurring at the workplace that could, or could not be simulated in real life (for example the blocking of a gauge) and are considering fuzziness of human reactions in unexpected situations. Figure 5 shows the main assessment steps.

We have followed 1000 subjects from construction industries during a twoyear period, developing a case base with 1000 safety use cases. The chosen subjects had a degree of decision power which gave them beyond the specific construction aptitudes also managerial ones; generally they were foremen, leaders of intervention teams, leaders of specific work teams, high degree skilled workers, which took the foremen place, in some small and medium enterprises, etc. Comparing these cases with the ideal (best case scenario) we have found the results presented in Table 1 .

It is possible to observe that more than $50 \%$ of the subjects were deviant from the good practice procedures. As main causes for this behaviour we found through a checklist completed by the subjects the following causes (the subjects were allowed to choose more than one cause).

Comparing bad behaviour against worst-case scenario using the HBFM framework we found the data presented in Table 3.

Bad behaviours (or traits) that were not accordingly HBFM were generally explained as very context specific- context that was not integrally modelled in HBFM worst-case scenario. 


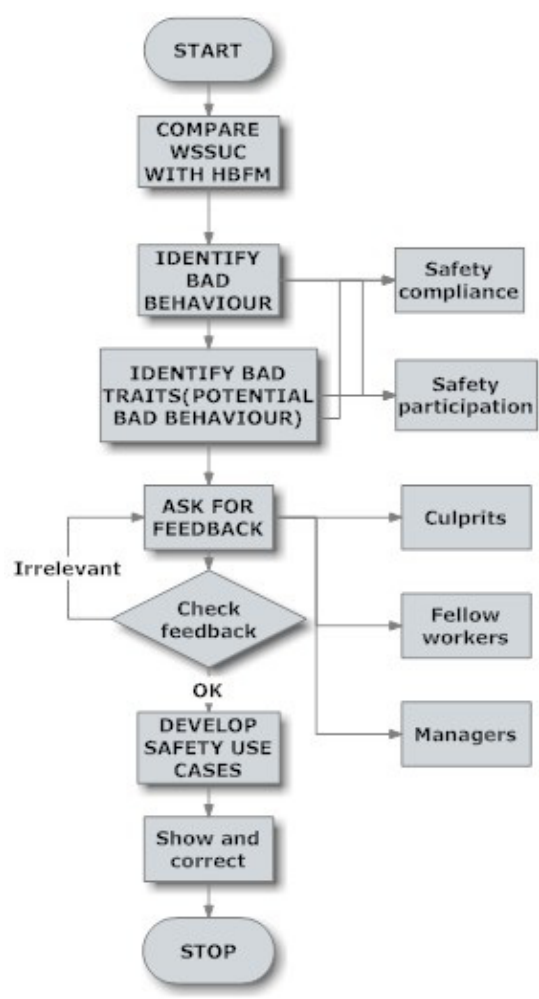

Figure 5: $\quad$ Main risk assessment steps.

Table 1: $\quad$ WSSUC vs. best-case HBFM.

\begin{tabular}{|c|c|c|}
\hline $\begin{array}{c}\text { Similarities } \\
\text { (over 70\% similar } \\
\text { traits) }\end{array}$ & $\begin{array}{c}\text { Slight deviations } \\
\text { (up to 55\% similar } \\
\text { traits) }\end{array}$ & $\begin{array}{c}\text { Significant deviations } \\
\text { (under 55\% similar } \\
\text { traits) }\end{array}$ \\
\hline $45 \%$ & $25 \%$ & $30 \%$ \\
\hline
\end{tabular}

Table 2: $\quad$ Major causes of deviation.

\begin{tabular}{|c|c|c|c|c|c|}
\hline $\begin{array}{c}\text { General } \\
\text { and } \\
\text { workplace } \\
\text { training }\end{array}$ & $\begin{array}{c}\text { Capabilities } \\
\text { (un)developed } \\
\text { at workplace }\end{array}$ & $\begin{array}{c}\text { Erroneous task } \\
\text { understanding }\end{array}$ & \multicolumn{3}{|c|}{$\begin{array}{c}\text { State of readiness (physical and } \\
\text { psychical) }\end{array}$} \\
\cline { 3 - 6 } & & $10 \%$ & $\begin{array}{c}\text { Improper } \\
\text { self- } \\
\text { control }\end{array}$ & $\begin{array}{c}\text { Spontaneous } \\
\text { actions }\end{array}$ & Other \\
\hline $20 \%$ & $25 \%$ & $5 \%$ & $35 \%$ & $5 \%$ \\
\hline
\end{tabular}


Table 3: $\quad$ WSSUC vs. worst-case HBFM.

\begin{tabular}{|c|c|c|}
\hline Similarities & \multicolumn{2}{|c|}{ Deviations } \\
\hline & $\begin{array}{c}\text { Explainable } \\
\text { deviations } \\
\text { (through } \\
\text { feedback) }\end{array}$ & $\begin{array}{c}\text { Unexplainable } \\
\text { deviations }\end{array}$ \\
\hline $65 \%$ & $25 \%$ & 10 \\
\hline
\end{tabular}

Analysing the behavioural patterns vs. the HBFM model we found some general types of behaviour taking into account the context:

- normal behaviour;

- reactive behaviour (generally when some of the results of the work are not compliant with the specification);

- mitigation behaviour (generally when a small incident was simulated or had really occurred- incident about which the operator knows and knows also how to handle it);

- panic behaviour (an unknown incident have occurred and the operator knows nothing about how to handle it).

\section{Conclusions}

The method of assessment presented in this paper is targeted upon the human factor being the main cause of incidents and accidents. In developing the method we have started looking to fundament our HBFM model on ontology for interpretations of specific behaviours, understanding ontology as:

- a set of concepts required for behaviour analysis in a systemic way;

- a base for the development of a hierarchical framework structure;

- a set of relationships between the main players at the workplace; here is defined also causality;

- definition of axioms; ontology could be considered as meta-level knowledge.

We have developed an ontology called OTME based on the four elements of the work system Operator-Task-Machine-Environment (Work Environment). The ontology served as a basis of the development of the Human Behavioural Fault Model (HBFM). HBFM is based on the OTME ontology that was developed using Protégé [11] development tool. HBFM and the risk assessment associated could be the start-up of a behavioural safety process, with the following main steps: access, design, train and re-train, start the process, extend the feedback and involvement process, enhance recognition and celebration [12].

HBFM is incorporating also the main aspects of the Safety Culture Maturity Model developed by the Keil Centre [13]. 
It supposes a sometimes lengthy observation of the persons involved in the supposed risk prone activities. However, this could be a useful thing because the results are very helpful for the safety practitioner:

- a Human Behaviour Failure Model specific for each economic activity which could be taken into account as a reference for pattern matching;

- individual behaviour patterns which could be:

- OK, being used in this case as a heuristic experience to improve other situation;

- wrong and could be corrected just through training and individual practice.

We found that once developed an incorrect capability (for example to speed up the work but not lowering the protection visor) this capability could be very quickly changed if the worker learns the possible consequences of his actions.

The developed behavioural clusters could be anyhow used as case study material, contributing to the improvement of the safety at work.

Potential incidents could be predicted and prevented. For example we found that persons who had the task to assure the lockout of the installation during a maintenance activity had a tendency to distract his attention looking at a window in a building nearby. A small worst case scenario in which the installation was put accidentally under tension was shown to the supervisor of the lockout and he understood his duties. The method is especially useful at workplaces where the worker is not changed frequently so his behavioural habits are relatively well defined through time.

The originality of our research resides, essentially, in developing and using a multi-knowledge, ontology based support in order to develop a human behavioural reference model framework. This model could be transformed and adapted in order to develop specific best case and worst case or intermediary scenarios that are describing the possible behaviour of the human operator in a specific activity at a specific moment; the pattern resulted from such a scenario is matched against the real pattern, observed from the worker over a period of time, pattern included in a worker specific safety use case (WSSUC). The outcomes of our research are the efficient behaviour improvement of those with an improper behaviour through showing them what could happen if they are continuing (immediate re-training sessions) and also the optimal continuous safety training using examples and models.

\section{References}

[1] Yoshino K., Construction of human error prediction and causality model and evaluation study of prediction characteristics, Research Material of the Central Research Institute of Electric Power Industry No. 95901, August 1995, Japan

[2] Romanian Labour Inspection Statistics, 2007

[3] Schmidt B., The modelling of human behaviour, Erlangen: SCS Publications, 2000, ISBN 1-565-55211-3 
[4] Goerger S.R., Validating Human Behavioral Models for Combat Simulations Using Techniques for the Evaluation of Human Performance, DoD 2005

[5] Parminter T., Porteous T.,Design of environmental strategies by policy agencies using human behavioural models, www.socialsystems.co.nz

[6] Galata Aph., Johnson N., Hogg D., Learning Behaviour Models of Human Activities, in Proceedings of BMVC99

[7] Andhra University M.A Social Work Paper-III: Dynamics of Human Behaviour Question paper, 2008, http://www.indiastudychannel.com/ exams/ExamPaper39305.aspx

[8] Introduction to Models and Methods of Understanding Human Behaviour,www.a6ba.com

[9] M.L. Minsky (Ed) Matter, minds and models, in Semantic Information Processing, MIT Press, Cambridge MA, 1968

[10] Legaspi R. et al, Cluster -based predictive modelling to improve pedagogic reasoning, in Computers in Human Behaviour, March Elsevier 2007

[11] http://protege.stanford.edu/

[12] Grainne A.M., Sween T.M., Improve your Safety Program with a Behavioural Approach, in Quality Safety Edge http:/qualitysafetyedge.com/ articles

[13] www.keilcentre.co.uk 